\title{
Sex-influenced Association of Nonalcoholic Fatty Liver Disease with Coronary Heart Disease
}

\author{
Mary F. Feitosa ${ }^{a,{ }^{*}}$, Alexander P. Reiner ${ }^{b}$, Mary K. Wojczynskia $^{\mathrm{a}}$, Mariaelisa Graff ${ }^{\mathrm{c}}$, Kari E. \\ North ${ }^{d}$, Jeffrey J. Carre ${ }^{\mathrm{e}}$, and Ingrid B. Borecki ${ }^{\mathrm{a}}$ \\ aDivision of Statistical Genomics, Department of Genetics, Washington University School of \\ Medicine, Saint Louis, MO \\ ${ }^{b}$ Department of Epidemiology, University of Washington, Seattle, WA \\ 'Department of Epidemiology, University of North Carolina, Chapel Hill, NC \\ ${ }^{d}$ Carolina Center for Genome Sciences, University of North Carolina, Chapel Hill, NC \\ eUniversity Health Sciences Image Lab, Wake Forest University, Winston Salem, NC
}

\begin{abstract}
Objective-This study investigated whether nonalcoholic fatty liver disease (NAFLD) predicts prevalent coronary heart disease (CHD).

Methods-Epidemiologic studies have used various definitions for NAFLD. Here, we considered both liver fat burden measured by CT (FL) and the non-specific measure of hepatic inflammation -alanine aminotransferase (ALT). The association of FL and ALT with CHD (self report of coronary bypass, myocardial infarction, or percutaneous transluminal coronary angioplasty) was investigated in 2,756 European-American participants of the Family Heart Study.
\end{abstract}

Results-FL ( $\mathrm{p}=0.0084)$ and ALT ( $\geq 40 \mathrm{U} / \mathrm{L}, \mathrm{p}=0.014)$ were each individually associated with prevalent CHD. However, when accounting for traditional metabolic risk factors in a multivariate model FL had no predictive value for CHD in either men or women; whereas ALT was a significant predictor of CHD in men, and the association strengthened among non-diabetic men. In non-diabetic women, neither FL nor ALT was associated with CHD.

Conclusions-ALT ( $\geq 40 \mathrm{U} / \mathrm{L}$ ) was a predictor of prevalent CHD in men but not in women, while CT measured FL was not significant in either sex. The failure to account for traditional risk factors, heterogeneity by sex, and varying definitions of NAFLD may account for some of the conflicting evidence in the literature regarding the association between NAFLD and coronary disease.

\footnotetext{
(C) 2013 Elsevier Ireland Ltd. All rights reserved.

"Correspondent author: Mary Furlan Feitosa, Ph. D., Division of Statistical Genomics, Washington University School of Medicine, 4444 Forest Park Blvd; St. Louis, MO, 63108-2212, Campus Box 8506, USA, Phone: (314) 747.3792; Fax: (314) 362.4227, mfeitosa@wustl.edu.

5. Disclosures

None.

Supplementary material

Supplementary data related to this article can be found online

Publisher's Disclaimer: This is a PDF file of an unedited manuscript that has been accepted for publication. As a service to our customers we are providing this early version of the manuscript. The manuscript will undergo copyediting, typesetting, and review of the resulting proof before it is published in its final citable form. Please note that during the production process errorsmaybe discovered which could affect the content, and all legal disclaimers that apply to the journal pertain.
} 


\section{Keywords}

cardiovascular disease; nonalcoholic steatohepatitis; sex-specific association; insulin resistance; glucose metabolism

\section{Introduction}

Nonalcoholic fatty liver disease (NAFLD) is characterized by increased triglyceride deposition in the liver in the absence of excessive alcohol intake or other known causes of liver disease [1-3]. The clinico-pathologic spectrum of NAFLD ranges from simple steatosis to nonalcoholic steatohepatitis (NASH) to cirrhosis $[1,4]$. While liver biopsy is the diagnostic reference standard for NAFLD, it is invasive and used infrequently in epidemiologic research. Non-invasive imaging such as unenhanced computerized tomography (CT) can accurately measure liver fat burden with low attenuation in the hepatic tissue, with $82 \%$ sensitivity and $100 \%$ specificity for detecting $>30 \%$ hepatic steatosis [5-6]. Alternatively, liver function tests such as alanine transaminase (ALT) levels are sometimes used to screen for liver function abnormalities. Elevated serum levels of ALT are indicative of liver damage, and have been used as a biochemical marker of hepatic inflammation to monitor the course and severity of NAFLD $[4,7-8]$ and as a surrogate marker of NAFLD in several epidemiologic studies [4,7-8].

NAFLD is the most common cause of abnormal liver function, ranging in prevalence from 10 to $40 \%$ in the general population of various countries and is far more prevalent among individuals with obesity and type 2 diabetes, occurring in 70\%-90\% in such patients [1-3, 9]. NAFLD is closely related to insulin resistance and can be considered part of the 'metabolic syndrome' [10-11]. The pathogenesis of NAFLD includes fatty acid lipotoxicity from lipolysis of dietary or visceral fat, insulin resistance, increased inflammation, oxidative stress and lipid peroxidation. Coronary heart disease (CHD) is related to several of these factors, including abdominal and overall obesity and insulin resistance, and it shares several additional risk factors with NAFLD including hypertension and dyslipidemia [11-14]. What is not clear is whether NAFLD independently influences risk of CHD. Using ALT levels as a surrogate measure of NAFLD, some studies found an association with cardiovascular risk $[4,8,12]$, while other studies failed to detect an independent association with cardiovascular outcomes [15]. ALT levels have low specificity $[1,4,8]$ to diagnose NAFLD which may have contributed, at least in part, to these discrepant conclusions.

A growing number of studies have addressed the relationship of NAFLD to prevalent and incident clinical coronary artery disease in subjects of European ancestry, though the evidence is mixed (reviewed in $[1,4,12,14,16])$. Differences in definition or diagnostic criteria for NAFLD and study patient characteristics (including presence of type 2 diabetes and other components of metabolic syndrome) may account for some of the conflicting results among studies. Also, the increased cardiovascular risk in patients with NAFLD might reflect the coexistence of underlying metabolic syndrome risk factors, which makes it difficult to distinguish the pathologic factors underlying the increased risk of coronary artery disease with NAFLD. Alternatively, NAFLD itself might confer a cardiovascular risk above and beyond that associated with other traditional risk factors, such as the components of metabolic syndrome.

In this study, we investigate whether NAFLD is an independent predictor of CHD. To address this question we test: (i) whether liver fat burden measured by CT, and hepatic inflammation measured by elevated ALT levels ( $\geq 40 \mathrm{U} / \mathrm{L}$ ) are associated with prevalent CHD; (ii) whether NAFLD is a predictor of CHD independent of their shared metabolic risk 
factors; and (iii) whether association modification by sex and/or diabetic status exists. We carried out this study in 2,756 European American (EA) participants of the NHLBI Family Heart Study (FamHS).

\section{Material and Methods}

\subsection{Study Design and Sample}

The Family Heart Study (https://dsgweb.wustl.edu/fhscc/) recruited 1,200 families, half randomly sampled, and half selected because of an excess of CHD or risk factor abnormalities as compared with age- and sex-specific population rates [17], from four population-based parent studies: the Framingham Heart Study, the Utah Family Tree Study, and two centers for the Atherosclerosis Risk in Communities study (Minneapolis, and Forsyth County, NC). Study participants belonging to the largest pedigrees were invited to undergo CT imaging for coronary artery calcification; and scans were further assessed to quantitative hepatic and abdominal fat burden. The description of CT scan measurements is presented in Supplemental Material available online.

A total of 2,756 subjects in 510 extended random and high CHD risk families were studied. Informed consent was obtained from all participants, and this project was approved by the Institutional Review Boards of all participating institutions.

\subsection{Phenotypes and Covariates}

Prevalent CHD was obtained by self report of coronary bypass, myocardial infarction, and/ or any of the following: coronary angioplasty, balloon angioplasty, atherectomy, stent, percutaneous transluminal coronary angioplasty, or percutaneous coronary intervention; the information was validated by hospital records.

The CT liver attenuation (LA) was measured by three round regions of interest (ROI's) in two slices of the superior right lobe of the liver. The six ROI's values (three from each slice) were averaged. Instead of a spleen standard, we used a standardized phantom at a density of water $(\mathrm{HU}=0)$ as well as increasing densities of calcium for standardization. Lower LA values indicate higher fat content. If the liver attenuation on unenhanced CT scans is $40 \mathrm{HU}$, the predicted hepatic steatosis is approximately $30 \%[1,5]$. We inverted the scale of LA by multiplying each value by negative one to produce a phenotype of fatty liver (FL) for which higher values are associated with greater liver fat.

Hepatic inflammation was assessed by levels of alanine transaminase (ALT in units of U/L). ALT levels were measured by reaction rate assay based on the conversion of NADH to NAD using the Vitros analyzer (Johnson \& Johnson Clinical Diagnostics, Inc. Rochester NY 14650). We discretized ALT $\geq 40 \mathrm{U} / \mathrm{L}$ to indicate hepatic inflammation in our models [18]. Insulin resistance was assessed by HOMA-IR (Homeostatic Model Assessment: fasting glucose $(\mathrm{mmol} / \mathrm{L}) *$ fasting insulin $(\mathrm{mU} / \mathrm{L}) / 22.5)$, and diabetes was defined as taking antihyperglycemic medication and/or fasting glucose $\geq 126 \mathrm{ml} / \mathrm{dl}$. Overall adiposity was characterized by body mass index $\left(\mathrm{BMI}, \mathrm{kg} / \mathrm{m}^{2}\right)$. Lipids were assessed by standard ultracentrifugation, and low density lipoprotein cholesterol (LDL-C) was calculated using the Friedewald formula [19]. Systolic blood pressure (SBP) and diastolic blood pressure (DBP) were taken three times using a random zero sphygmomanometry in a sitting position and the mean of the second and the third measurements was used. A log transformation of HOMA-IR was taken to better approximate normality.

Information on antihyperglycemic, antihypertensive and lipid-lowering medications, current cigarette smoking (all measures as: $0=\mathrm{No}, 1=\mathrm{Yes}$ ) and on habitual alcohol intake were obtained by questionnaire. The clinic interviewer asked whether the subject "presently 
drinks alcoholic beverages at all (alcohol intake: $0=\mathrm{No}, 1=$ Yes)" and if so, the number of $1.5 \mathrm{oz}$ cocktails, $12 \mathrm{oz}$ glasses (or can) of beer, and $4 \mathrm{oz}$ glasses of wine they consumed per day in 1 week. Based on these responses, total alcohol intake (g/day) was calculated. Subjects were excluded from the analyses if: (i) alcohol consumption exceeded 21 drinks/ week for men and >14 drinks/week) for women; (ii) if taking Amiodarone, or (iii) if positive for hepatitis C antibodies. In all, 88 subjects for FL and 124 subjects for ALT were excluded.

\subsection{Statistical analysis}

Groups were compared using t-test for continuous variables and $\chi^{2}$ test for categorical variables. For association analysis, we employed a mixed model (proc glimmix using SAS ${ }^{\circledR}$ ) to account for the non-independence of family members according to their kinship using a logistic link function. Age, age ${ }^{2}$, sex, current cigarette smoking (No/Yes), alcohol intake (No/Yes) and clinical centers were included in each model of CHD. A p value of less than 0.05 for covariates was considered statistically significant in the association model.

\section{Results}

\subsection{Characteristics of the Study Population by CHD Status}

Table 1 shows the descriptive statistics of European American participants in the FamHS with and without CHD. The prevalence of CHD was significantly higher in men (18.9\%) as compared to women (6.2\%). On average, the subjects with CHD were 12.5 years older than unaffected subjects. The mean levels of FL, BMI, HOMA-IR and SBP were significantly higher with among those with CHD compared to those without CHD (Table 1). The mean levels of LDL-C and DBP were lower in subjects with CHD compared to those without, which may be attributable to the differential proportion of subjects taking medications: $62 \%$ of CHD subjects (vs. 27\% in non-CHD) were taking antihypertensive medications and $67 \%$ took lipid-lowering medications ( $V S .18 \%$ in non-CHD).

\subsection{Univariate Associations with Prevalent CHD}

First, we tested whether FL or ALT levels ( $\geq 40 \mathrm{U} / \mathrm{L}$ ) individually were associated with prevalent CHD (Table 2). Both FL ( $\mathrm{p}=0.0084$ ) and ALT levels ( $\geq 40 \mathrm{U} / \mathrm{L}, \mathrm{p}=0.014)$ were predictors of CHD. We also found the expected associations between CHD and traditional risk factors, such as diabetes ( $\mathrm{p}<0.0001)$, HOMA-IR $(\mathrm{p}<0.0001), B M I(\mathrm{p}=0.012)$, LDL-C $(\mathrm{p}<0.0001)$, SBP $(\mathrm{p}=0.047)$ and DBP $(\mathrm{p}<0.0001)$.

\subsection{Multivariate Associations of Prevalent CHD with Metabolic Traits}

We sought to determine whether NAFLD has an independent association above and beyond that explained by traditional risk factors, namely, obesity, insulin resistance, and diabetes. We fit a multivariate model including FL and ALT with these other CHD risk factors. When accounting for the metabolic factors, which increase risk for both NAFLD as well as CHD, the marginal association of FL ( $\mathrm{p}=0.598$ ) was no longer significant (Table 3), suggesting that the association observed in the univariate model could be explained by these common underlying factors. However, ALT ( $\geq 40 \mathrm{U} / \mathrm{L}, \mathrm{p}=0.042$ ) remained associated with prevalent CHD. Since the prevalence of CHD was much higher among men (18.8\%) compared to women $(6.2 \%)$, we further investigated whether the association results differed by sex (Table 3). An independent association with ALT ( $\geq 40 \mathrm{U} / \mathrm{L}, \mathrm{p}=0.044$ ) was noted but for men only.

Since diabetic status and use of hyperglycemic medications represent a potential source of heterogeneity, we tested the robustness of these associations while restricting the analysis to non-diabetic subjects (Supplementary Table 1). The same pattern of results was obtained. 
However the significance of ALT ( $\geq 40 \mathrm{U} / \mathrm{L}, \mathrm{p}=0.007$ ) as a predictor in men was enhanced, lending additional credibility to the conclusion that this indicator of hepatic inflammation is an independent predictor of CHD risk in men; we continued to note no association in women.

We then expanded the predictive model to include several other variables that are utilized in the Framingham risk score for CHD (http://www.framinghamheartstudy.org/risk/ coronary.html) such as lipids (LDL-C) and blood pressure measurements (SBP and DBP). Since the effects of lipid-lowering medications and antihypertensive medications may influence the results, we also included these indicators as covariates in the multivariate model (Supplementary Table 2). The results were qualitatively robust and similar to the previous analysis (Supplementary Table 1), again showing a sex-specific association of ALT ( $\geq 40 \mathrm{U} / \mathrm{L})$ with $\mathrm{CHD}(\mathrm{p}=0.031)$ independent of traditional CHD risk factors in non-diabetic men.

\section{Discussion}

Diverse findings for the relationship of NAFLD with atherosclerosis and clinical coronary artery disease have been reported in the literature, some studies supporting NAFLD as a cardiovascular risk factor [14, 20-22], others not [23, reviewed in 4, 8, 14-16]. There may be several reasons that could affect the different conclusions reached by these studies. On the phenotypic level, NAFLD represents a wide spectrum of liver damage, and differences in definition or diagnostic criteria for NAFLD may contribute to conflicting results; notably, some studies defined NAFLD on the basis of ALT levels alone [e.g., 8, 21]. Also, the coexistence of underlying metabolic risk factors in subjects with NAFLD (e.g., diabetes and insulin resistance) makes it difficult to distinguish whether NAFLD per se is a predictor of CHD, or whether the common metabolic risk factors are responsible for the association with CHD. Few previous studies examined the heterogeneity of NAFLD by sex. In this study, we set out to investigate whether NAFLD, characterized by either FL or ALT levels ( $\geq 40 \mathrm{U} / \mathrm{L}$ ), is an independent predictor of prevalent CHD, while simultaneously modeling the common metabolic risk factors and allowing for sex differences. Even though elevated ALT levels are not diagnostic of NAFLD in practice, they are often regarded as a harbinger of liver disease or a marker of severity. In this context, our data provides support for elevated ALT levels (indicative of non-specific liver damage) being associated with increased risk of $\mathrm{CHD}$, at least in men, which is more readily appreciated when considering non-diabetic subjects. Thus, there are aspects of liver disease that do appear to independently exacerbate risk for CHD, although those factors differ in men and women, and appear to be confounded by underlying insulin resistance and diabetes.

Much remains unknown about the complex mechanisms underlying the association of NAFLD with atherosclerosis and coronary artery disease, though insulin resistance, oxidative stress, inflammatory markers (e.g., IL-6, TNF- $a$ and CRP), obesity-related ectopic fat (in nonadipose tissues) and lipotoxicity appear to be important interacting factors [12, 24]. Here, we observed that the association between CT measured FL with CHD was attenuated by adjustment for insulin resistance and diabetes in the multivariate model. This suggests that the association of FL with CHD may be mediated via insulin resistance and diabetes. In a study of diabetic patients, liver fat measured by ultrasound was a predictor of prevalent cardiovascular disease independent of traditional risk factors [25]. NAFLD is very common in diabetic patients [25], consistent with the idea that insulin resistance is an early manifestation common to both the accumulation of liver fat and to diabetes, and a strong CHD risk factor. 
Hepatic inflammation, indicated by elevated ALT levels, may influence risk of coronary artery disease. Our study showed that ALT ( $\geq 40 \mathrm{U} / \mathrm{L}$ ) predicts prevalent CHD independent of traditional cardiovascular risk factors, such as BMI, insulin resistance, diabetes, LDL-C, SBP and DBP in men, but not in women. A similar observation was made in the Hoorn Study, in which association between ALT and CHD remained significant for subjects in the third tertile relative to those in the first tertile after adjustment for traditional risk factors [8]. Another study found that increased ALT activity was associated with risk of CHD as estimated using the Framingham risk score in both sexes; however, the ALT threshold for an increased risk of CHD was higher in men (> 43 IU [26]). In addition, serum gammaglutamyltransferase levels, which have been used as an index of liver dysfunction, were associated with all-cause cardiovascular mortality in men (but not in women), independent of other cardiometabolic risk factors [22]. Previous literature has shown that increased ALT was a predictor of coronary calcification [20] and related with the risk of carotid intima medial thickness in patients with NAFLD [21], independent of risk factors for atherosclerosis and metabolic syndrome. Moreover, severity of liver histopathology (degree of hepatic steatosis, necroinflammation, and fibrosis) was strongly associated with early carotid atherosclerosis, even controlling for traditional risk factors, in subjects with NAFLD [14].

Systemic up-regulation of inflammatory activity may simultaneously influence NAFLD, coronary atherosclerosis and CHD [24]. Indeed, patients with these diseases have higher plasma markers of oxidative stress and inflammation [12, 16, 24]. One possible mechanism of up-regulation of inflammatory activity is via hepatic insulin resistance [24], which is associated with NAFLD [12, 16, 24]. Hepatic insulin resistance is characterized by a reduced insulin-suppressing effect on glucose production in the liver, which exacerbates peripheral insulin resistance and hyperinsulinemia [24]. This leads to increased hepatic free fatty acid synthesis and decreased synthesis of apolipoprotein B-100, resulting in accumulation of triglycerides in the liver [12, 24]. It has been suggested that hepatic insulin resistance is mediated by free fatty acids and tumor necrosis factor-alpha (TNF-a) through JNK (c-Jun N-terminal kinase)-dependent insulin receptor substrate-1 phosphorylation. The

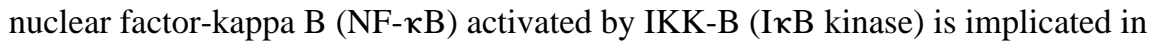
transcription of pro-inflammatory mediators (e.g., interleukin-1 (IL-1), interleukin-6 (IL-6) and TNF- $a$ ). NF- $\kappa B$ dependent inflammatory mediators produced in hepatocytes downregulate insulin sensitivity and trigger liver injury $[12,24]$. These same proinflammatory cytokines and pathways are activated during the process of atherosclerotic plaque formation $[12,16,24]$, which may play a dual role in the development of NAFLD and coronary disease.

This study has several relevant strengths and limitations. It is a large population-based study in which imaging data characterizing liver attenuation was available along with measurements of all other major cardiovascular risk factors. While it is not possible to characterize subjects with histology requiring liver biopsies in otherwise healthy subjects, we have an unprecedented opportunity to investigate associations among these conditions in a large epidemiologic study. CT imaging of the liver provides a non-invasive procedure that produces an accurate characterization of fat burden $[1,4,5]$. ALT levels have been the most commonly used variable for assessment of liver disease [18]. However, elevated ALT levels are not specific to NAFLD and can occur in response to many other conditions and, in that sense, they are not an adequate measure of NAFLD and they do not reflect the severity or the outcome of NAFLD [1, 4]. ALT measurement often fails to identify patients with minimal to mild necroinflammatory activity and normal liver enzymes may be observed in NAFLD [4]. The sensitivity of ALT for the diagnosis of NASH in large cohort studies was found to be only $40 \%$ [4], and there is no consensus on a standard cut-off value for detecting the presence of NASH [8]. However, several previous epidemiological studies have used 
ALT levels to define NAFLD [7, 8]. Even if ALT levels do not specifically indicate NAFLD, it appears that the level of hepatic inflammation indicated by ALT (for whatever reason) predict prevalent CHD. We have used HOMA-IR as a measure of insulin resistance, which correlates reasonably well $\left(\mathrm{r}^{2}=-0.67, \mathrm{p}<0.001\right.$ [27] $)$ with the results of hyperinsulinemic euglycemic glucose clamp technique. Although this technique is a gold standard for assessment of insulin sensitivity, the complexity of this methodology limits its use in epidemiologic studies [27]. We used prevalent CHD as an outcome, and prospective data would be preferable in this context. The lack of association between NAFLD traits and CHD in the FamHS women may be due to the relatively young age of women and perhaps they have not had sufficient time to manifest CHD. It is worth mentioning that the presentation of coronary artery disease in women tends to be 10 years later than its presentation in men [28]. Nevertheless, other larger populations should investigate whether NAFLD predicts clinical cardiovascular events in women. Lastly, because half of the families were selected by an excess of CHD our findings should be considered in similar populations.

In summary, advanced hepatic inflammation may have an independent effect promoting CHD. A mechanism involving markers of oxidative stress and inflammation [12, 15, 16, 24] may play a crucial role in the progression of FL to histological changes associated with steatohepatitis and simultaneously in advancing development of coronary artery disease. Our findings suggest that, ALT levels ( $\geq 40 \mathrm{U} / \mathrm{L}$ ) predict CHD in men, while association of FL with CHD may be mediated via insulin resistance and diabetes. The sex-specific associations observed here are novel and reinforce the idea that the metabolic profiles of men and women influence risk of CHD differently. The mechanisms underlying these associations merit further investigation.

\section{Supplementary Material}

Refer to Web version on PubMed Central for supplementary material.

\section{Acknowledgments}

This study was supported by the by R01-DK-8925601 and R01-DK-075681 (Ingrid B. Borecki, PI) from NIDDK, and R01-HL-087700 and R01-HL-088215 (Michael A. Province, PI) from NHLBI.

\section{References}

1. Lewis JR, Mohanty SR. Nonalcoholic fatty liver disease: a review and update. Dig Dis Sci. 2010; 55:560-78. [PubMed: 20101463]

2. Mulhall BP, Ong JP, Younossi ZM. Non-alcoholic fatty liver disease: An overview. J Gastroenterol Hepatol. 2002; 17:1136-43. [PubMed: 12453271]

3. Angulo P. Nonalcoholic fatty liver disease. N Engl J Med. 2002; 346:1221-31. [PubMed: 11961152]

4. Byrne CD, Olufadi R, Bruce KD, Cagampang FR, Ahmed MH. Metabolic disturbances in nonalcoholic fatty liver disease. Clin Sci (Lond). 2009; 116:539-64. [PubMed: 19243311]

5. Kodama Y, Ng CS, Wu TT, Ayers GD, Curley SA, Abdalla EK, Vauthey JN, Charnsangavej C. Comparison of CT methods for determining the fat content of the liver. AJR Am J Roentgenol. 2007; 188:1307-12. [PubMed: 17449775]

6. Boyce CJ, Pickhardt PJ, Kim DH, et al. Hepatic steatosis (fatty liver disease) in asymptomatic adults identified by unenhanced low-dose CT. AJR Am J Roentgenol. 2010; 194:623-8. [PubMed: 20173137]

7. Yamada J, Tomiyama H, Yambe M, et al. Elevated serum levels of alanine aminotransferase and gamma glutamyltransferase are markers of inflammation and oxidative stress independent of the metabolic syndrome. Atherosclerosis. 2006; 189:198-205. [PubMed: 16405892] 
8. Schindhelm RK, Diamant M, Dekker JM, Tushuizen ME, Teerlink T, Heine RJ. Alanine aminotransferase as a marker of non-alcoholic fatty liver disease in relation to type 2 diabetes mellitus and cardiovascular disease. Diabetes Metab Res Rev. 2006; 22:437-43. [PubMed: 16832839]

9. Browning JD, Szczepaniak LS, Dobbins R, et al. Prevalence of hepatic steatosis in an urban population in the United States: impact of ethnicity. Hepatology. 2004; 40:1387-95. [PubMed: 15565570]

10. Hamaguchi M, Kojima T, Takeda N, et al. The metabolic syndrome as a predictor of nonalcoholic fatty liver disease. Ann Intern Med. 2005; 143:722-8. [PubMed: 16287793]

11. Marchesini G, Bugianesi E, Forlani G, et al. Non-alcoholic fatty liver, steatohepatitis, and the metabolic syndrome. Hepatology. 2003; 37:917-23. [PubMed: 12668987]

12. Treeprasertsuk S, Lopez-Jimenez F, Lindor KD. Nonalcoholic fatty liver disease and the coronary artery disease. Dig Dis Sci. 2011; 56:35-45. [PubMed: 20464495]

13. Hamaguchi M, Kojima T, Takeda N, et al. Nonalcoholic fatty liver disease is a novel predictor of cardiovascular disease. World J Gastroenterol. 2007; 13:1579-84. [PubMed: 17461452]

14. Targher G, Day CP, Bonora E. Risk of cardiovascular disease in patients with nonalcoholic fatty liver disease. N Engl J Med. 2010; 363:1341-50. [PubMed: 20879883]

15. Fraser A, Harris R, Sattar N, Ebrahim S, Smith GD, Lawlor DA. Gamma-glutamyltransferase is associated with incident vascular events independently of alcohol intake: analysis of the British Women's Heart and Health Study and Meta-Analysis. Arterioscler Thromb Vasc Biol. 2007; 27:2729- 35. [PubMed: 17932318]

16. Nseir W, Shalata A, Marmor A, Assy N. Mechanisms Linking Nonalcoholic Fatty Liver Disease with Coronary Artery Disease. Dig Dis Sci. 2011; 56:3439-49. [PubMed: 21655948]

17. Higgins M, Province M, Heiss G, Eckfeldt J, et al. NHLBI Family Heart Study: objectives and design. Am J Epidemiol. 1996; 143:1219-28. [PubMed: 8651220]

18. Giannini EG, Testa R, Savarino V. Liver enzyme alteration: a guide for clinicians. CMAJ. 2005; 172:367-79. [PubMed: 15684121]

19. Friedewald WT, Levy RI, Fredrickson DS. Estimation of the concentration of low-density lipoprotein cholesterol in plasma without the use of the preparative ultracentrifuge. Clin Chem. 1972; 18:499. [PubMed: 4337382]

20. Jung DH, Lee YJ, Ahn HY, Shim JY, Lee HR. Relationship of hepatic steatosis and alanine aminotransferase with coronary calcification. Clin Chem Lab Med. 2010; 48:1829-34. [PubMed: 20961204]

21. Wang CC, Lin SK, Tseng YF, et al. Elevation of serum aminotransferase activity increases risk of carotid atherosclerosis in patients with non-alcoholic fatty liver disease. J Gastroenterol Hepatol. 2009; 24:1411-6. [PubMed: 19702910]

22. Haring R, Wallaschofski H, Nauck M, Dorr M, Baumeister SE, Volzke H. Ultrasonographic hepatic steatosis increases prediction of mortality risk from elevated serum gamma-glutamyl transpeptidase levels. Hepatology. 2009; 50:1403-11. [PubMed: 19670414]

23. McKimmie RL, Daniel KR, Carr JJ, et al. Hepatic steatosis and subclinical cardiovascular disease in a cohort enriched for type 2 diabetes: the Diabetes Heart Study. Am J Gastroenterol. 2008; 103:3029- 3035. [PubMed: 18853970]

24. Malaguarnera M, Di Rosa M, Nicoletti F, Malaguarnera L. Molecular mechanisms involved in NAFLD progression. J Mol Med (Berl). 2009; 87:679-95. [PubMed: 19352614]

25. Targher G, Bertolini L, Padovani R, et al. Prevalence of nonalcoholic fatty liver disease and its association with cardiovascular disease among type 2 diabetic patients. Diabetes Care. 2007; 30:1212-8. [PubMed: 17277038]

26. Ioannou GN, Weiss NS, Boyko EJ, Mozaffarian D, Lee SP. Elevated serum alanine aminotransferase activity and calculated risk of coronary heart disease in the United States. Hepatology. 2006; 43:1145-51. [PubMed: 16628637]

27. Hung AM, Sundell MB, Egbert P, et al. A comparison of novel and commonly-used indices of insulin sensitivity in African American chronic hemodialysis patients. Clin J Am Soc Nephrol. 2011; 6:767-74. [PubMed: 21441124] 
28. Lawton JS. Sex and gender differences in coronary artery disease. Semin Thorac Cardiovasc Surg. 2011; 23:126-30. [PubMed: 22041042] 


\section{Research highlights}

We investigated whether NAFLD is a predictor of CHD independent of traditional risk factors.

NAFLD was assessed by using CT measured fatty liver and elevated levels of alanine aminotransferase levels (ALT $\geq 40 \mathrm{U} / \mathrm{L}$ ).

We investigated whether association modification by sex exists between NAFLD traits and prevalent CHD.

Elevated ALT levels ( $\geq 40 \mathrm{U} / \mathrm{L}$ ) are associated with increased risk of CHD in men only, and the significance of ALT as a predictor is enhanced in non-diabetic subjects.

Association of liver fat burden measured by CT with CHD may be mediated via insulin resistance and diabetes. 


\section{Table 1}

Characteristics of European American participants by Presence or Absence of CHD in the FamHS

\begin{tabular}{lccc}
\hline & with CHD & without CHD & P value \\
\hline Sample size & 327 & 2,429 & \\
Men & 233 & 1003 & $<.0001$ \\
Age (years) & $68.1 \pm 9.1$ & $55.6 \pm 13.1$ & $<.0001$ \\
FL (HU) & $-57.5 \pm 11.6$ & $-59.4 \pm 11.1$ & 0.0047 \\
FL $\geq-40 \mathrm{HU}^{*}\left(\mathrm{~N}_{\text {Total }} ; \%\right.$ of affected) & $25(311 ; 8.0)$ & $152(2,328 ; 6.5)$ & 0.056 \\
ALT $\geq 40 \mathrm{U} / \mathrm{L}^{*}\left(\mathrm{~N}_{\text {Total }} ; \%\right.$ of affected $)$ & $24(312 ; 7.7)$ & $138(2,314 ; 6.0)$ & 0.046 \\
BMI $\left(\mathrm{kg} / \mathrm{m}^{2}\right)$ & $29.8 \pm 5.1$ & $28.7 \pm 5.7$ & 0.0004 \\
HOMA-IR & $4.10 \pm 4.85$ & $2.64 \pm 2.46$ & $<.0001$ \\
Diabetes $\left(\mathrm{N}_{\text {Total }} ; \%\right.$ of affected $)$ & $103(327 ; 31.5)$ & $263(2,427 ; 10.8)$ & $<.0001$ \\
LDL-C (mg/dL) & $92.1 \pm 30.3$ & $113.3 \pm 33.4$ & $<.0001$ \\
Lipid-lowering medication $\left(\mathrm{N}_{\text {Total }} ; \%\right.$ of affected) & $207(308 ; 67.2)$ & $420(2,334 ; 18.0)$ & $<.0001$ \\
SBP (mm Hg) & $125.6 \pm 22.4$ & $120.2 \pm 19.6$ & $<.0001$ \\
DBP (mm Hg) & $68.3 \pm 10.1$ & $70.0 \pm 9.8$ & 0.0055 \\
Antihypertensive medication $\left(\mathrm{N}_{\text {Total }} ; \%\right.$ of affected) & $203(327 ; 62.1)$ & $655(2,425 ; 27.0)$ & $<.0001$ \\
\hline
\end{tabular}

Variables are shown as number $(\mathrm{N})$, and/or frequency $(\%)$, or mean \pm standard deviation;

$\mathrm{P}$ values refer to t-test for continuous variables and $\chi^{2}$ test for categorical variables;

FL: Fatty liver (liver attenuation * -1); ALT: levels of alanine transaminase; BMI: body mass index; HOMA-IR: Homeostatic Model Assessment (fasting glucose (mmol/L)*fasting insulin (mU/L)/22.5); Diabetes: taking hyperglycemic medication and/or fasting glucose $\geq 126 \mathrm{ml} / \mathrm{dl}$ ); LDL-C: low-density lipoprotein cholesterol; SBP: systolic blood pressure; SBP: diastolic blood pressure;

*Fisher's Exact Test (table probability) 
Table 2

Univariate Association of CHD with NAFLD and Other Metabolic Traits

\begin{tabular}{lccc}
\hline Independent Trait & Odds Ratio Estimate $(\mathbf{9 5 \%}$ CI) & P value & N Total $($ CHD) \\
\hline FL & $1.116(1.043 ; 1.329)$ & 0.0084 & $2639(311)$ \\
ALT $\geq 40 \mathrm{U} / \mathrm{L}$ & $2.014(1.156 ; 3.509)$ & 0.014 & $2626(312)$ \\
BMI & $1.033(1.007 ; 1.060)$ & 0.012 & $2753(327)$ \\
HOMA-IR & $1.109(1.062 ; 1.157)$ & $<.0001$ & $2693(318)$ \\
Diabetes & $2.582(1.870 ; 3.566)$ & $<.0001$ & $2754(327)$ \\
LDL-C & $0.979(0.973 ; 0.984)$ & $<.0001$ & $2642(308)$ \\
SBP & $0.993(0.986 ; 1.015)$ & 0.047 & $2752(327)$ \\
DBP & $0.961(0.947 ; 0.976)$ & $<.0001$ & $2752(327)$ \\
\hline
\end{tabular}

ALT $\geq 40 \mathrm{U} / \mathrm{L}$ defines abnormality. 
